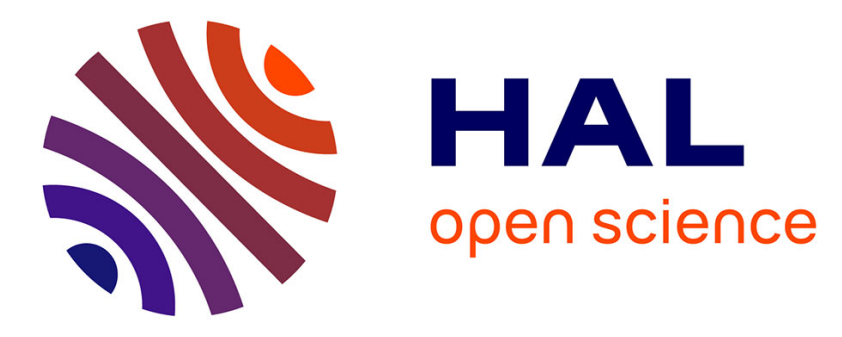

\title{
A Modified Parametric Estimation of ultrasound Doppler Velocity
}

Denis Kouamé, Jean-Pierre Remenieras, Jean-François Roux, Abdeldjalil Ouahabi, Marc Lethiecq

\section{- To cite this version:}

Denis Kouamé, Jean-Pierre Remenieras, Jean-François Roux, Abdeldjalil Ouahabi, Marc Lethiecq. A Modified Parametric Estimation of ultrasound Doppler Velocity. IEEE International Instrumentation and Measurement Technology Conference, Jun 1996, Brussels, Belgium. pp.431-434, 10.1109/IMTC.1996.507420 . hal-03153377

\section{HAL Id: hal-03153377 \\ https://hal.science/hal-03153377}

Submitted on 26 Feb 2021

HAL is a multi-disciplinary open access archive for the deposit and dissemination of scientific research documents, whether they are published or not. The documents may come from teaching and research institutions in France or abroad, or from public or private research centers.
L'archive ouverte pluridisciplinaire HAL, est destinée au dépôt et à la diffusion de documents scientifiques de niveau recherche, publiés ou non, émanant des établissements d'enseignement et de recherche français ou étrangers, des laboratoires publics ou privés. 
IEEE Instrumentation and Measurement

Technology Conference

Brussels, Belgium, June 4-6, 1996

\title{
A Modified Parametric Estimation of ultrasound Doppler Velocity
}

\author{
D. Kouamé, J.P. Remenieras, J.F. Roux, A. Ouahabi, M. Lethiecq. \\ LUSSI, GIPULTRASONS / EIT \\ 7 avenue Marcel Dassault 37004 Tours Cedex. FRANCE \\ Phone : (+33) 47711216 or 0666 Fax: (+33) 47279533 E-mail: kouame@balzac.univ-tours.fr
}

\begin{abstract}
A new recursive least square (RLS) method is here used to compute complex ARMA in a two-step Doppler flow velocity estimate. Compared with the classical RLS case, this method provides better results, and needs few additional computations. This method is applied to the Doppler ultrasound flow velocity measurement through frequency estimates. Thus, after the computation of the complex AR parameters of the Doppler analytic signal in a first step, we propose in a second step, a direct velocity measurement by using these parameters, without an explicit computation of the Power Spectral Density (PSD). This method compared with the PSD peaks detection is more accurate and almost independant of the Signal-to-Noise Ratio.
\end{abstract}

\section{INTRODUCTION}

Many methods have been expiored to estimate flow speeds in Doppler systems. In real-time two-dimensional (2-D) Doppler systems, the velocity estimate is done with few samples (8-16) in comparison with the image cadence. This has implied the development of fast algorithms with low accuracy. In the case of precise measurements of flow velocities, it is necessary to find adapted methods.

The ultrasound Doppler flow velocity measurements of afluid are linked to the estimate of a specific trequency referred to as "Doppler frequency". In fact, the precise determination of this frequency is not easy because of different physical parameters which introduce uncertainty in the velocity estimation [11].

To overcome the inconvenients of the use of the classical fast Fourier Transform (which leads to bad precision when only few samples are available or when SNR is (ow), a number of investigations have been performed with ARMA parametric identification. Most studies dealing with these methods use algorithms applied on block data, assuming that the Doppler signal is almost stationary on a time window. The choice of the time window, to avoid hiding non stationarities and keeping a sufficient number of samples, is critical. In practice a compromise based on experimental observations is made to define this time length of window.

The Doppler frequency is then estimated by mean of the power spectral density (PSD). This can be performed either by implicit or explicit centroid computing (first order moment) or by detecting PSD amplitude peaks. Even if in the same conditions the peaks detection is better, the estimates - in any case are biased, especially when SNR is low and Doppler bandwidth is large. Their computations can also be very heavy.

We present an approach which directly computes the Doppler frequency using more precise (compared with classical RLS case) AR parameters estimates.

\section{THE RECURSIVE LEAST SQUARE (RLS) ALGORITHM}

We first define some useful notations. Let $\mathrm{x}$ be a function of time $k$, and $T$ the sampling period.

$$
\begin{aligned}
& \rho x(k)=\frac{\Delta(k+1)-x(k)}{T} \\
& \int_{0}^{\tau} x(v)=T \sum_{i=0}^{k} x(i)
\end{aligned}
$$

A signal $x$ can be described by an ARMA model:

Let us consider the invariant ARMA model so that

$$
A(\rho) x(t)=B(\rho) u(t)+\eta(t)
$$

with $\left\{\begin{array}{l}A(\rho)=\rho^{n}+a_{n-1} \rho^{n-1}+\ldots+a_{0} \\ B(\rho)=b_{m} \rho^{m}+\ldots+b_{0}\end{array}\right.$

where $x$ is the output of the modeled system, $u(t)$ and $\eta(t)$ are non-correlated centered white noises.

Assuming that 


$$
\left\{\begin{array}{l}
\theta=\left[\begin{array}{llll}
-a_{n-1} & \ldots-a_{0} & b_{m} \ldots b_{0}
\end{array}\right]^{\top} \\
\phi(t)= \\
{\left[\begin{array}{l}
\rho^{n-1} x(t) \rho^{n-2} x(t) \ldots x(t) \rho^{m_{u}} u(t) \ldots u(t)
\end{array}\right]^{H}} \\
y(t)=\rho^{n} x(t)
\end{array}\right.
$$

The model (3) is equivalent to

$y(t)=\phi^{H}(t) \theta+\eta(t)$

where $\mathrm{H}$ denotes the hermitian conjugate

The estimation $\hat{\theta}$ of $\theta$ in the least square sense lies on the calcution of the cost function:

$$
\begin{aligned}
J(\hat{\theta}, t) & =\frac{1}{2}\left\{\frac{1}{C} S_{0}^{t}\left[y(v)-\phi^{H}(v) \hat{\theta}(t)\right]^{2}\right. \\
& \left.+\left(\hat{\theta}(t)-\hat{\theta}\left(t_{0}\right)\right)^{H} P_{0}^{-1}\left(\hat{\theta}(t)-\hat{\theta}\left(t_{0}\right)\right)\right\}
\end{aligned}
$$

where $t_{0}$ is an instant before, but close to $t$. This means that in discrete time $\mathrm{t}_{0}=(\mathrm{k}-1) \mathrm{T}$ and $\mathrm{t}=\mathrm{kT}$ for example. In the classical RLS estimation, $\hat{\theta}\left(t_{0}\right)$ is constant during the computations. This condition is discarded here, and adaptive values are used.

$P_{0}$ is a non-negative symmetrical matrix - like $P$ below which can be chosen as diagonal to simplify the computations; $\mathrm{C}$ is a positive constant which can make it possible to limit the fluctuations in the estimates.

The best estimate $\hat{\theta}$ follows from the minimum of $\mathrm{J}$, by the cancellation of its gradient. After some simple, but long computations, the recursive estimates of $\theta$ can be expressed by:

$$
\begin{aligned}
& \rho \hat{\theta}(t)=P(t)\left\{P_{0}^{-1} \rho \hat{\theta}\left(t_{-1}\right)+\frac{\phi(t)}{C}\left[y(t)-\phi^{H}(t) \hat{\theta}\left(t_{0}\right)\right]\right\} \\
& \rho P(t)=-\frac{P(t) \phi(t) \phi H_{(t) P(t)}}{C+T_{\phi}^{\top}(t) P(t) \phi(t)}
\end{aligned}
$$

by choosing $t_{-1}$ similarly to $t_{0}$, with $t_{-1}<t_{0}$ (for example $\left.\mathrm{t}_{-1}=(\mathrm{k}-2) \mathrm{T}\right)$

In the classical derivation the term $P_{0}^{-1} \rho \hat{\theta}(t-1)$ doesn't exist.

The advantage of our algorithm is that it computes at each step of recursion the increment of estimated parameters, and it uses the best knowledge of the process.

Figure $1 \mathrm{a}$ and $1 \mathrm{~b}$ compare the parameters estimates of the following model : $y(k)=-(0.0625+0.5 i) y(k-1)+(1+i) y(k-1)+$ $(3+5 i) e(k-3)+(2+2 i) e(k-2)-1.5 e(k-1)+e(k)$.

where $\eta$ is a white noise.

We only present the imaginary part $s$ of the parameters to reduce the number of figures; the real parts have the same behavior.

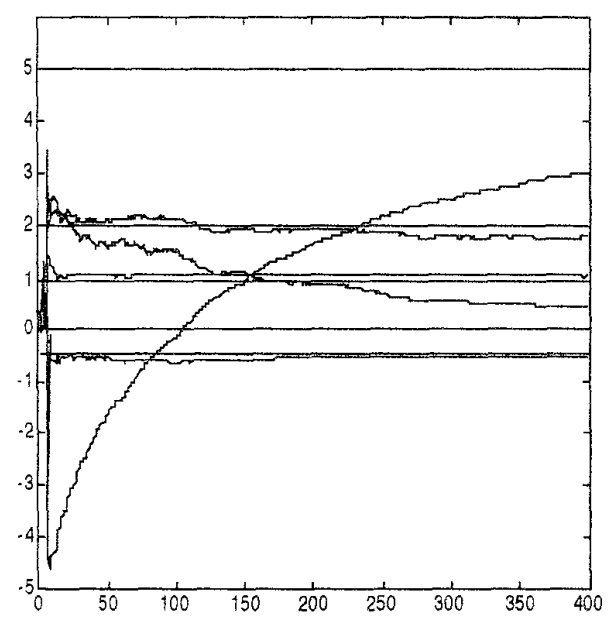

Fig. 1a. Imaginary parts of the parameters estimates of the ARMA model (10) with the classical RLS algorithm.

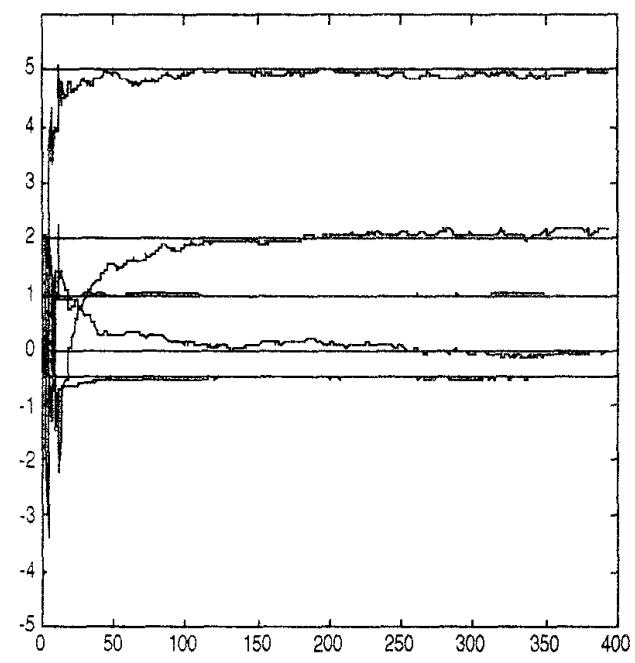

Fig. 1b. Imaginary part of the parameters estimates of the ARMA model (10) with the new approach 


\section{THE SPECTRAL DECOMPOSITION}

The Doppler frequency estimate lies on the calculation of the Power Spectral Density (PSD) which is defined in the case of ARMA model by:

$P(f)=\gamma\left|\frac{\sum_{i=1}^{m} b_{1} \exp (-2 \pi j l f T)}{\sum_{k=1}^{n} a_{k} \exp (-2 \pi j k f T)}\right|^{2}$

where $\gamma$ is a constant depending on the covariance of the modelling error.

One of the best ways [8], [11] of determining the Doppler frequency is to search the PSD amplitude peak's frequency.

Thus, this procedure inludes three steps: the first one is the computation of ARMA or AR parameters estimation, the second one is the computation of the PSD, and finally the estimation of the peak frequency.

Young and Song [5] proposed a direct estimation of the centroid (instead of the peak frequency) by using an AR2 $(n=2$ and $m=0$ in (4)). They considered that the two frequencies are characteristic of the Doppler signal (Doppler frequency and clutter).

in pratice this is not always right. And because of many sources of noises there are more than two frequencies. So, the question is which of them is the Doppler frequency. We thus perform a decomposition of the PSD into simple first order elements. In the rest of the paper we only consider AR model (but the procedure can also be applied to ARMA models).

Let us first consider a first order AR model $(n=1)$ or AR1.

The peak frequency is given by: $\mathrm{f}_{\mathrm{pl}}=\frac{\alpha_{1}}{2 \pi \mathrm{T}}$

where $\alpha_{1}>0$ is the argument of $a_{1}$.

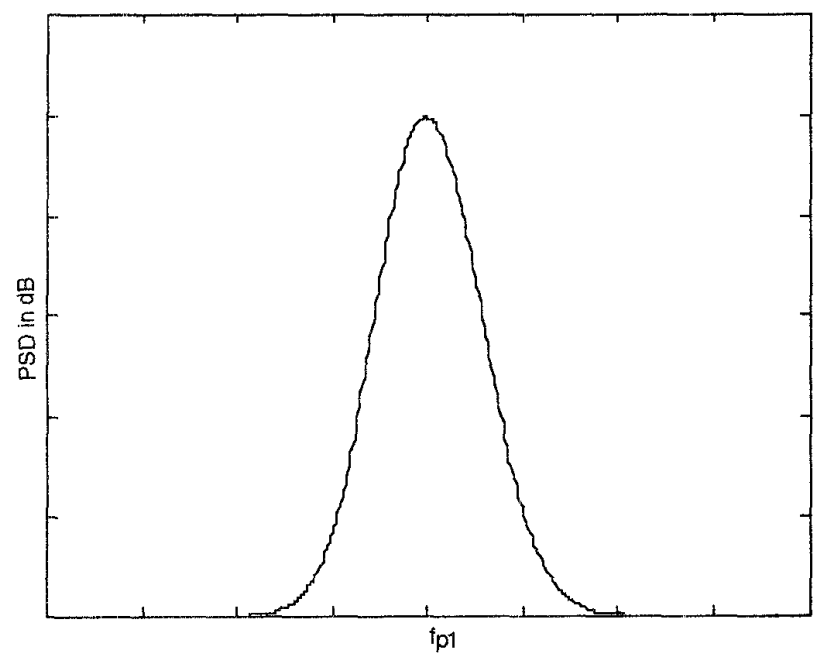

Fig. 2a. PSD of an AR1

Now, let us consider an ARn. Since the denominator of (11) is a n-order complex polynom it can be expressed as a product of first order polynoms. Thus in this case the PSD can be expressed as:

$$
P(f)=\frac{\gamma}{\left|\prod_{k=1}^{n}\left(z-z_{k}\right)\right|_{z=\exp (-2 \pi j \mathrm{j} T)}^{2}}
$$

where the $z_{k}$ are the roots of the denominator of (11). Thus if its logarithm is taken, the PSD is the sum of first order elements; and the peaks frequencies $\mathrm{f}_{\mathrm{pk}}$ of these elements are expressed as in (12)

$$
\mathrm{f}_{\mathrm{pk}}=\frac{\alpha_{k}}{2 \pi \mathrm{T}}
$$

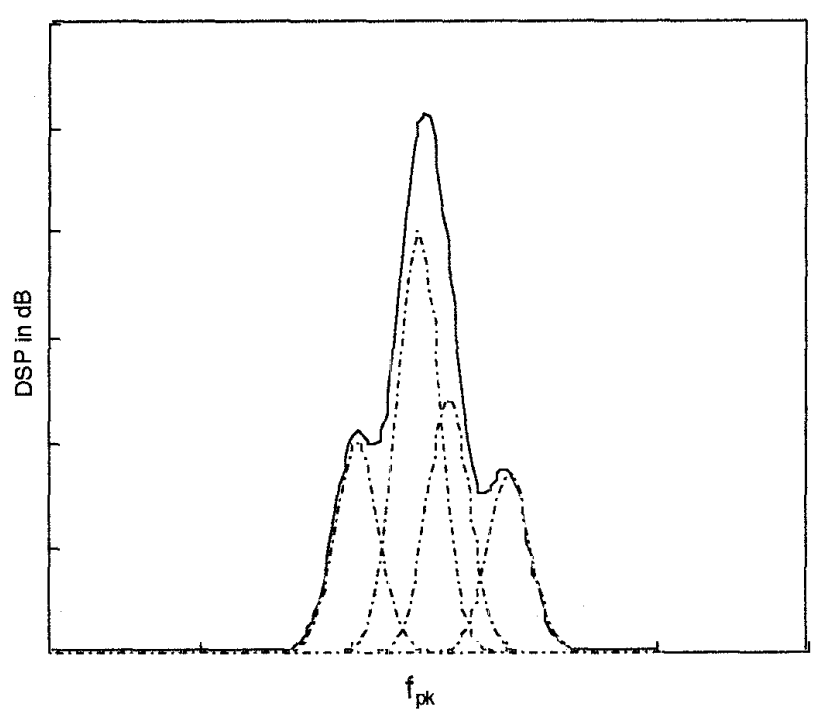

Fig.2b. PSD of an Arn model

With this decomposition we note that the minimum of $\alpha_{k}$ gives the best estimation of Doppler frequency. Thus the direct computation of the PSD is not necessary. In the case when there is a high level of clutter, it is required to make a test to distinguish the frequency caused by the Doppler effect and the one caused by the clutter.

This method compared with the amplitude peaks detection seems to be quite independant of signal-tonoise ratio (SNR), when the model described in [11] is used, as shown on fig. 3. On these figures, we show the speed (Doppler fequency) estimates: Fig $3 a$ and $3 b$. compare in the invariant speed case, the peaks estimate and the pole decomposition estimate with SNR $=0$ (fig. $3 a$ ) and $-20 \mathrm{~dB}$ (fig. $3 \mathrm{~b}$ ). It can be seen that the pole decomposition method is more accurate.

When the speed varies (fig. 4), it is impossible to make a good estimate with peaks method without averaging. wheras the decomposition method is still correct. 


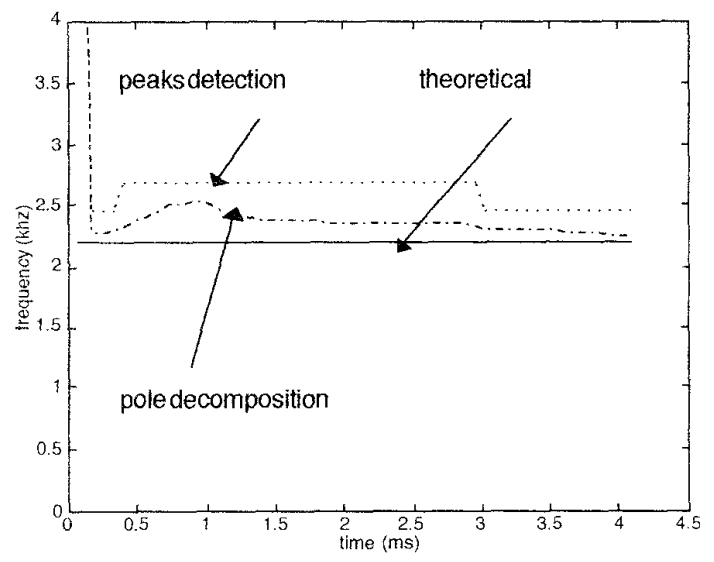

Fig. 3a. Doppler frequency estimate with $s n r=0 \mathrm{~dB}$; invariant speed case

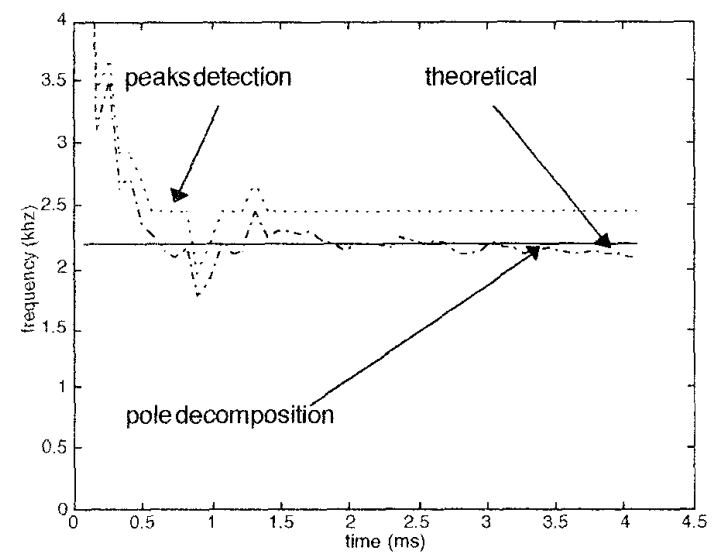

Fig. 3b. Doppler frequency estimate with $s n r=-20 \mathrm{db}$ invariant speed case

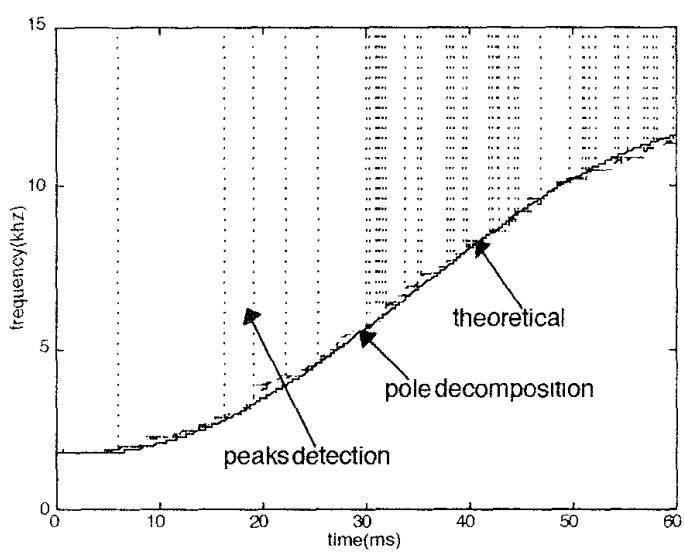

Fig. 4. Doppler frequency estimate with $s n r=10 \mathrm{db}$ time variant speed case

\section{CONCLUSION}

A transformated recursive least square algorithm is used here to estimate the parameters of an AR Doppler analytic signal. Through the PSD decomposition the Doppler frequency is directly obtained from the parameters onward. This Doppler frequency estimate compared to the peaks frquency approach is quite independant of SNR.

\section{REFERENCES}

[1] L.Ljung. "System identification". Theory of user. Prentice Hall. N.J.1987.

[2] S.L Marple, "Digital Spectral Analysis with applications ", Prentice Hall Signal Processing Series, N.J, 1987.

[3] G.C.Goodwin, R.H.Middleton. "Digital control and estimation. A unified approch". Prentice Hall 1990.

[4] Y.N.Chen, Y.C.Wu, "Modified recursive Least Square algorithm for parameter identification ", Int. J. of Science, vol23, n², pp187-205, 1992.

[5] Young Box Ahn and Song Bai Park, "estimation of Mean frequecy and variance of Ultrasonic Doppler Signal by using second order autoregressive model ". IEEE on ultrasonics, ferroelectrics and frequency control. Vol.38 $\mathrm{N}^{\circ} 3$, May 1991

[6] J.L.Garbini,F.K.Forster,J.E.Jorgensen:"Measure-ment of fluid turbulence based on pulsed ultrasound techniques. Part 1. Analysis. J.Fluid.Mech. vol.118,pp445-470",.1982,.

[7] J.L.Garbini, F.K.Forster, J.E Jorgensen "Measurement of fluid turbulence based on pulsed ultrasound techniques. Part 2 Experimental investigation." J. Fluid. Mech., vol.118, pp 471$505 ", 1982$

[8] F. Wendling, "Simulation of Doppler ultrasound signals, for laminar, pulsatile, non uniform flow", Master of science in the School of Mechanical Engineering , Georgia Institute of Technology, February 1991.

[9] W.D.Barber, J.W.Eberhard, S.G.Karr, "A new time domain technique for velocity measurements using Doppler ultrasound, IEEE Trans. Biomed. Eng., volBME-32,pp213-229,1985.

[10] K.Kristoffersen, "Time-Domain estimation of the center frquency and spread of Doppler spectra in Diagnostic ultrasound, "IEEE Trans. Ultraseon, Ferroelec. Freq. Control., vol. UFFC-35, no4, pp484-497, 1988.

[11] S. A. Jones and D. P. Giddens. "A simulation of transit time effects in Doppler ultrasound signals ". Uitrasound in Med. \& Biol. Vol 16, pp 607619,1990 